\title{
APLICAÇÃO DO MÉTODO SIMPLE AGGREGATION OF PREFERENCES EXPRESSED BY ORDINAL VECTORS - MULTI DECISION MAKERS (SAPEVO-M) PARA SELEÇÃO DE VIATURAS BLINDADAS DO CORPO DE FUZILEIROS NAVAIS (CFN) EM MISSÕES DE GARANTIA DA LEI E DA ORDEM (GLO)
}

\author{
Gabriel Henrique Fernandes \\ Centro Universitário Augusto Motta (UNISUAM) \\ Av. Paris, 84 - Bonsucesso, Rio de Janeiro/RJ \\ gabrielhfernandes@hotmail.com
}

Roberta Anastácia Palermo Fernandes

Universidade Estadual da Zona Oeste (UEZO)

Av. Manuel Caldeira de Alvarenga, 1203 - Campo Grande, Rio de Janeiro/RJ

roberta_anas@hotmail.com

\section{Marcos dos Santos}

Centro de Análises de Sistemas Navais (CASNAV) / Instituto Militar de Engenharia (IME) Praça Barão de Ladário, Ilha das Cobras, Rua da Ponte, Ed. 23, Centro, Rio de Janeiro/RJ marcosdossantos doutorado@yahoo.com.br

\section{Luiz Frederico Horácio de Souza de Barros Teixeira}

Centro de Análises de Sistemas Navais (CASNAV)/ Universidade Federal Fluminense (UFF) Praça Barão de Ladário, Ilha das Cobras, Rua da Ponte, Ed. 23, Centro, Rio de Janeiro/RJ

frederico.horacio@gmail.com

\section{Carlos Francisco Simões Gomes}

Universidade Federal Fluminense (UFF)

Rua Passo da Pátria, 156 - 209, São Domingos, Niterói - RJ cfsg1@bol.com.br

\section{RESUMO}

Este artigo apresenta um estudo sobre a relevância da aplicação do método SAPEVO-M para auxiliar na escolha da melhor viatura blindada a ser utilizada em missões de Garantia da Lei e da Ordem. Este método (SAPEVO-M) tem por objetivo gerar pesos entre os critérios, nas quais as alternativas estão classificas, bem como a pontuação das alternativas nos critérios, a partir da opinião dos decisores, de forma ordinal. Cada critério tem seu peso adequado ao cenário que será aplicado, sendo este executado em missões especiais de GLO. Tendo o método SAPEVO-M como base para o desenvolvimento deste trabalho, foi utilizado o programa SapevoWeb com o intuito de compilar os dados dos critérios e as alternativas para a ordenação das mesmas. O objetivo da aplicação do método é buscar a alternativa que busque uma contribuição para o aprimoramento do sistema de apoio à decisão de uma unidade militar de blindados do Corpo de Fuzileiros Navais da Marinha do Brasil, e que favoreça eventuais missões operacionais na resolução de problemas relacionados a multicritérios de apoio a decisão a partir de sua notação matemática. A metodologia utilizada neste trabalho é de caráter exploratório, visto que se trata de um estudo de caso. Este estudo se iniciou com a realização de uma pesquisa, que contou com a participação de colaboradores operadores de blindados da Marinha do Brasil, buscando 
caracterizar este processo.

Palavra-chave: SAPEVO-M; SapevoWeb; Garantia da Lei e da Ordem; Corpo de Fuzileiros Navais; Apoio Multicritério à Decisão (AMD).

\begin{abstract}
This paper presents a study on the relevance of applying the SAPEVO-M method to assist in choosing the best armored vehicle to be used in Law and Order Assurance missions. This method (SAPEVO-M) aims to generate weights between the criteria, in which the alternatives are classified, as well as the score of the alternatives in the criteria, from the opinion of the decision makers, ordinally. Each criterion has its weight appropriate to the scenario that will be applied, which is performed in special GLO missions. With the SAPEVO-M method as the basis for the development of this work, the SapevoWeb program was used in order to compile the criteria data and the alternatives for their ordering. The objective of the application of the method is to look for the alternative that seeks a contribution to the improvement of the decision support system of a military unit of the Brazilian Navy Marine Corps, and that favors eventual operational missions in the resolution of related problems. the decision support multicriteria from its mathematical notation. The methodology used in this work is exploratory, since it is a case study. This study began with a research, which was attended by employees of the Brazilian Navy armored operators, seeking to characterize this process.
\end{abstract}

Keywords: SAPEVO-M; SapevoWeb; Guarantee of Law and Order; Marine Corps; Multicriteria Decision Aid (MCDA).

\title{
Como Citar:
}

FERNANDES, Gabriel Henrique; FERNANDES, Roberta Anastácia Palermo; SANTOS, Marcos dos; TEIXEIRA, Luiz Frederico Horácio de Souza de Barros; GOMES, Carlos Francisco Simões. Aplicação do Método Simple Aggregation Of Preferences Expressed By Ordinal Vectors - Multi Decision Makers (SAPEVO-M) para seleção de viaturas blindadas do Corpo de Fuzileiros Navais (CFN) em missões de Garantia da Lei e da Ordem (GLO). In: SIMPÓSIO DE PESQUISA OPERACIONAL E LOGÍSTICA DA MARINHA, 19., 2019, Rio de Janeiro, RJ. Anais [...]. Rio de Janeiro: Centro de Análises de Sistemas Navais, 2019.

\section{INTRODUÇÃO}

A segurança pública é um dos direitos coletivos da sociedade moderna prevista pela constituição Brasileira sob a responsabilidade do estado. Este, por sua vez, tem como dever a manutenção da ordem pública e a incolumidade da pessoa humana, sendo respeitado o direito de ir e vir do cidadão brasileiro.

O combate à criminalidade tem se tornado um dos maiores desafios para o estado, frente ao domínio das facções criminosas nas comunidades brasileiras. Tal fato reflete-se no aumento de operações dentro das comunidades de todo Brasil para intervir nas ações ilícitas praticadas dentro e fora das mesmas. As missões de Garantia da Lei e da Ordem (GLO) são realizadas exclusivamente por ordem expressa da Presidência da República, nos casos em 
que há o esgotamento das forças tradicionais de segurança pública, em graves situações de perturbação da ordem. Podem ser aplicadas em todo território nacional, onde se fizer necessário com a anuência do chefe do poder executivo federal. Segundo a Emenda Constitucional $n^{\circ} 99$ de 14 de dezembro de 2017, Art. 142:

As Forças Armadas (FFAA), constituídas pela Marinha do Brasil (MB), Exército Brasileiro (EB) e Aeronáutica (FAB), são instituições nacionais, permanentes e regulares, organizadas com base na hierarquia e na disciplina, sob autoridade suprema do Presidente da República, e destinam-se à defesa da Pátria, à garantia dos poderes constitucionais e, por iniciativa de qualquer destes, da lei e da ordem. (BRASIL, 1988)

Os militares das FFAA são treinados para estarem aptos a atender às diferentes demandas do Estado Brasileiro, em diversas situações. No que tange à segurança pública, as Forças Armadas podem, por exemplo, contribuir para o preparo e treinamento de agentes, fornecer dados de inteligência e apoio logístico, como suporte de pessoal, material e estruturas. Atualmente as FFAA vem usando seus carros blindados em operações de GLO, porém não há uma certeza sobre o melhor blindado para ser utilizado em cada uma destas ocasiões.

Ao iniciar um estudo de apoio multicritério à tomada de decisão (AMD) dentro de uma instituição, esta deve estar preparada para passar por mudanças e aceitar os desafios que serão gerados por este processo, que trará consigo não somente resoluções de problemas, mas também melhorias contínuas para a instituição em questão.

Diante do exposto, ter um projeto de AMD seria uma forma de robustecer as decisões, para que se possa traçar um plano focado em diminuir a ocorrência de falhas, reduzindo custos com retrabalho e perdas, melhorando a qualidade nos processos dos serviços prestados e assim atendendo de forma satisfatória às necessidades da missão a ser empregada. Este artigo foi dividido em Introdução, Descrição do Problema, Fundamentação Teórica, Modelagem Matemática, Resultados e Conclusão.

\section{DESCRIÇÃO DO PROBLEMA}

O presente trabalho trata-se de estudo realizado em uma Organização Militar (OM) dedicada ao treinamento de integração dos Grupamentos Operativos de Fuzileiros Navais por meio de veículos blindados e carros de combate, a fim de que tais grupamentos passem a ter maior poder de fogo, ampliação da capacidade de manobras, proteção e melhor desenvolvimento defensivo anticarro e, ainda, condições para efetuar ações de reconhecimento e de segurança. A organização vem atuando dentro desta finalidade do poder nacional há mais de quinze anos, visando: contribuir com ações de apoio a infantaria com os carros de combate, apoio à movimentação tática com transporte blindado e também com apoio logístico; integração de defesa anticarro; ser um elemento de manobra; e sob reforço, efetuar operações de reconhecimento, segurança, vigia e economia de forças.

Esta organização pertence ao Corpo de Fuzileiros Navais (CFN) e tem suas instalações localizadas no bairro da Ilha do Governador, Zona Norte do Rio de Janeiro. Uma das dificuldades desta OM é a escolha de carros de combate a serem usados em missões de GLO, que pode gerar custos extras e desperdícios, devido à incompatibilidade da viatura blindada com o tipo de missão que ela irá exercer.

Assim, este estudo visa responder a seguinte pergunta: dentre os atuais modelos em uso no CFN, qual a viatura blindada mais adequada para missões de GLO, levando em consideração a natureza da atividade e as características operacionais da viatura?

O método SAPEVO-M será usado para auxiliar o processo decisório. A fim de facilitar a visualização dos fatores relevantes do problema em questão, a Figura 1 apresenta 
um mapa mental com as condições de contorno do problema.

Figura 1: Condições de contorno do problema

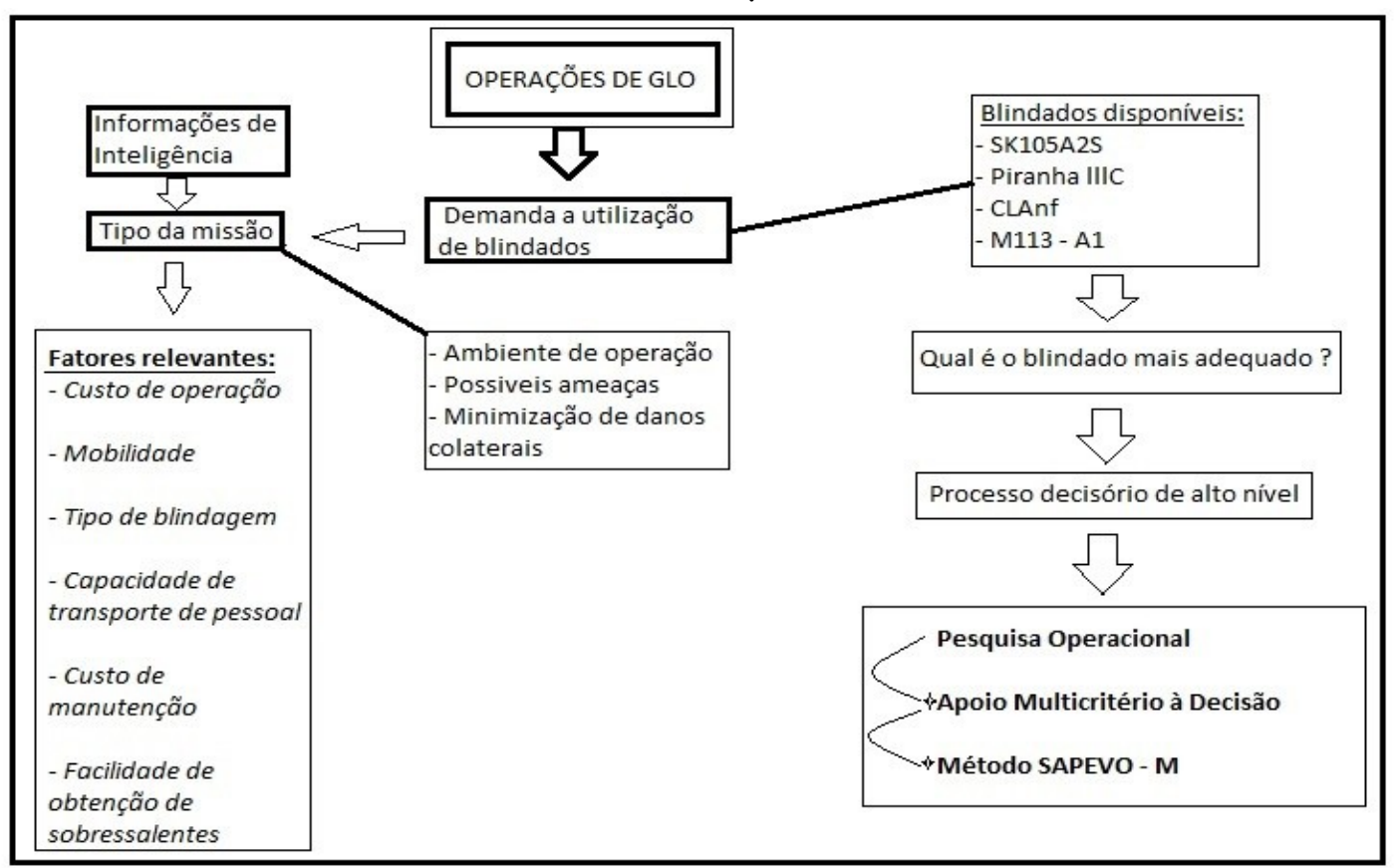

Fonte: Autores (2019)

\section{REFERENCIAL TEÓRICO}

\subsection{PESQUISA OPERACIONAL}

Segundo Arenales (2007), a Pesquisa Operacional (PO) pode proporcionar a melhoria no funcionamento de um sistema levando em conta o recurso da empresa. Desta forma, a PO busca em sua estrutura a solução ótima para a resolução dos problemas indicados.

Segundo Hillier e Lieberman (2006), a PO teve início na Segunda Guerra Mundial. Com envio de recursos para diversos lugares durante as operações bélicas, os planejadores observaram diferentes maneiras para conseguir seus objetivos táticos e formular novas estratégias. Ainda segundo os mesmos autores, com o progresso da tecnologia computacional, a pesquisa operacional obtém um crescimento significante, pois fica mais acessível tendo em vista a rapidez dos computadores em relação aos cálculos feitos a mão. Algumas técnicas utilizadas em pesquisa operacional são: Teoria dos Jogos, Teoria dos Grafos, Teoria das Filas, Teoria da Simulação, Programação Linear, Probabilidade e Estatística Matemática e Programação Dinâmica.

Segundo Marins (2011), o caminho do estudo da pesquisa operacional leva o profissional de PO a adquirir um raciocínio estruturado. Esta padronização do raciocínio contribui para a interpretação e análise dos problemas reais, como pode-se observar na Figura 2. 


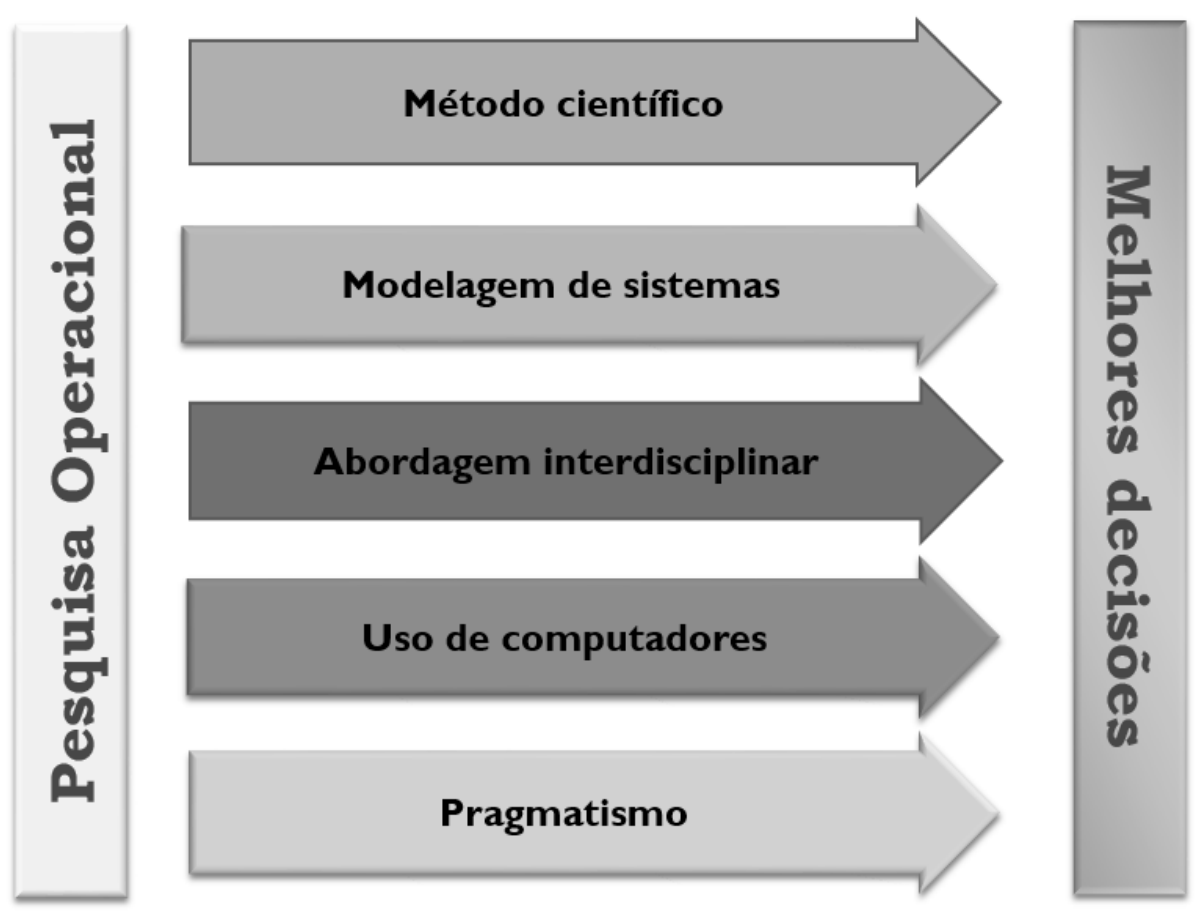

Figura 2: Pesquisa Operacional. Fonte: Site da SOBRAPO (2019)

\subsection{MÉTODOS DE APOIO MULTICRITÉRIO À DECISÃO}

Nas últimas décadas observou-se um crescimento na disponibilização de informações e a dificuldade de se usar as mesmas nos processos de decisão. A partir disso, surgiram diversos métodos com o intuito de contribuir com os agentes decisores com uma maior objetividade nas tomadas de decisão.

Os métodos multicritérios de apoio à decisão são técnicas de assessoramento ao agente decisor (indivíduo, grupo de indivíduos, comitê de especialistas ou lideranças) para a tomada de decisões sobre problemas complexos, avaliando, ordenando ou selecionando alternativas mediante diferentes pontos de vista e dentro de critérios pré-definidos (GOMES e GOMES, 2019).

Segundo Brans e Mareschal (2005) o surgimento dos métodos multicritérios de tomada de decisão, são considerados ferramentas com base matemática, capazes de resolver problemas em que há critérios conflitantes.

De Almeida (2013) afirma que, todos os dias, diversas decisões são tomadas nas organizações. Além disso, cada decisão leva em consideração múltiplas alternativas analisadas sob múltiplos critérios, daí a importância de se dispor de um cabedal de métodos capazes de estruturar a subjetividade do processo decisório.

\subsection{MÉTODO SAPEVO-M}

Segundo Gomes, Mury e Gomes (1997) o método Simple Aggregation of Preferences Expressed by Ordinal Vectors (SAPEVO) ou, em tradução livre, agregação simples de preferência expressas por vetores ordinais, é um método simples de ser aplicado. Isto ocorre porque só é necessário que o decisor realize uma comparação paritária entre os critérios, e, posteriormente, entre as alternativas dentro de cada critério, apenas por relações de preferência. Assim, o método SAPEVO desdobra o problema decisório a partir de três etapas básicas, quais sejam: 
- $1^{0}$. Transforma as preferências ordinais dos critérios em um vetor de pesos de critérios;

- $\quad 2^{\circ}$ Transforma as preferências ordinais de alternativas para um dado conjunto de critérios de classificação em pesos parciais de alternativas e

- $3^{\circ}$. Determina os pesos globais das alternativas.

O método SAPEVO-M (Simple Aggregation of Preferences Expressed by Ordinal Vectors - Multi Decision Makers) é uma evolução do método SAPEVO original, consistindo num método ordinal para problemas tipicamente do tipo P.ү, isto é, cujo resultado pretendido é um procedimento de ranking. Esta nova versão estendeu o emprego do método para múltiplos decisores. Além disso, introduziu uma verificação de consistência, mediante o processo de normalização da matriz (TEIXEIRA et al., 2019).

A transformação das preferências ordinais de critérios em um vetor de pesos de critérios ocorre de maneira que, dado um conjunto de critérios de classificação, estes são comparados em pares. Isto produz uma matriz D das alternativas gerais $\delta_{i j}$ tais que:

$$
\begin{aligned}
& \delta_{i j}=1 \leftrightarrow i \approx j(1) \\
& \delta_{i j}>1 \leftrightarrow i>j \delta_{i j} \gg 1 \leftrightarrow i>>j \\
& \delta_{i j}<1 \leftrightarrow i<j \delta_{i j} \ll 1 \leftrightarrow i \ll j(3)
\end{aligned}
$$

onde:

₹ é tão importante quanto.

$>$ é mais importante que.

》 é muito mais importante que.

Cabe destacar que, embora o método SAPEVO-M seja um método ordinal, isto é, para o estabelecimento de um ranking, Gomes e Maia (2012) atestam que este tipo de método também é comumente empregado em problemas de seleção, cujo resultado pretendido é uma escolha.

\section{MODELAGEM MATEMÁTICA}

\subsection{ALTERNATIVAS DE CARRO DE COMBATE}

Para o desenvolvimento do trabalho foram selecionados quatro modelos de carros blindados, que são detalhados a seguir: 


\subsubsection{CARRO DE COMBATE SK105A2S}

O Carro de Combate Leve Sobre Lagartas (CCL SL), Steyr Sk105A2S Kurassier, é fabricado na Áustria e foi adquirido e incorporado ao CFN no ano de 2001. Atualmente está alocado na unidade do Batalhão de Blindados de Fuzileiros Navais. O Kurassier é um veículo blindado, correntemente utilizado como equipamento de apoio ao combate pela sua ação de choque em favor da tropa apoiada. Com seu tanque cheio, referente a 310 litros de Diesel, este carro de combate consegue ter um raio de ação de $520 \mathrm{~km}$. Possui a capacidade de comportar somente sua própria tripulação, não realizando transporte de pessoal. Em termos de dimensão, sua blindagem é de $40 \mathrm{~mm}$, seu tamanho é de 7,76 x 2,50 m (comprimento x largura), tendo velocidade máxima de $70 \mathrm{~km} / \mathrm{h}$. A Figura 3 apresenta o modelo do carro blindado.

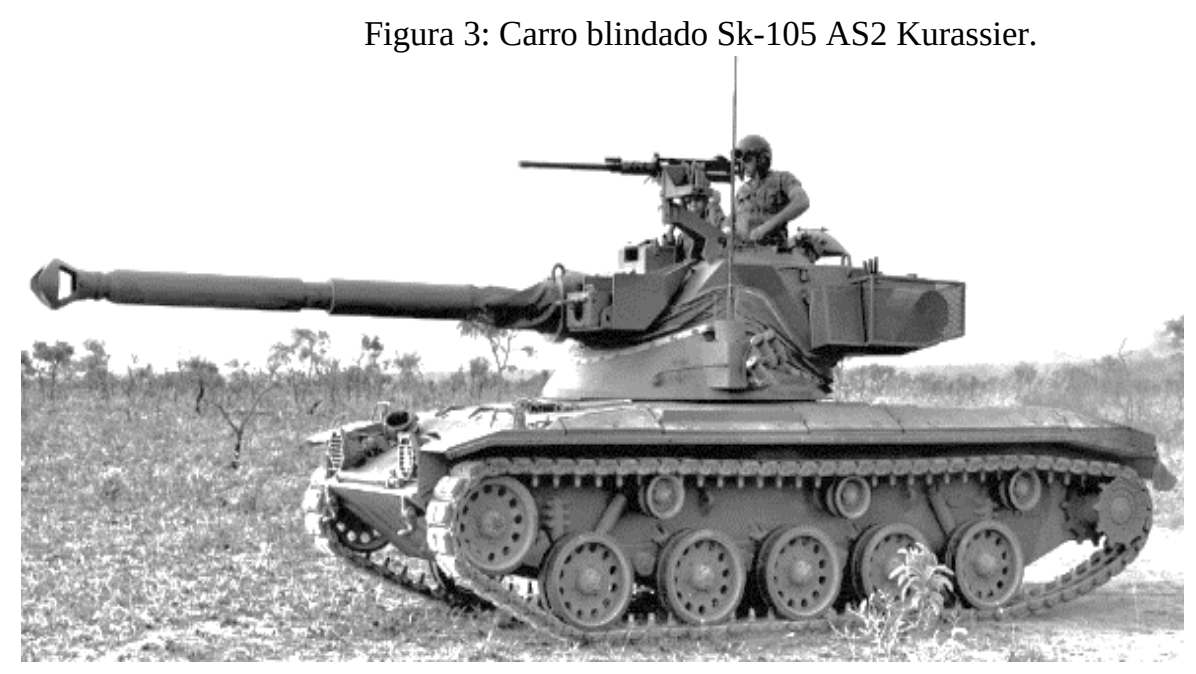

Fonte: Brasil (2008).

\subsubsection{PIRANHA IIIC}

A viatura blindada sobre rodas Piranha IIIC, é um dos carros mais bem-sucedidos dentro da família de veículos blindados sobre rodas. Esse modelo de veículo tem se apresentado com grande capacidade de adaptação aos diferentes tipos de terreno. Seu emprego pode ser em um simples transporte de pessoal ou até mesmo o combate propriamente dito. Com seu tanque cheio referente a 300 litros de diesel, recebe um raio de ação de $780 \mathrm{~km}$. Possui capacidade total de transporte de 14 militares, sendo que duas dessas vagas são para o operador do veículo e chefe do carro e 12 fuzileiros com seus equipamentos. Em termos de dimensão, sua blindagem é de $40 \mathrm{~mm}$, seu tamanho é de 7,30 x 2,66 m (comprimento x largura), podendo atingir velocidade máxima de $100 \mathrm{~km} / \mathrm{h}$. Esse blindado pode ser observado na Figura 4. 
Figura 4: Piranha IIIC.

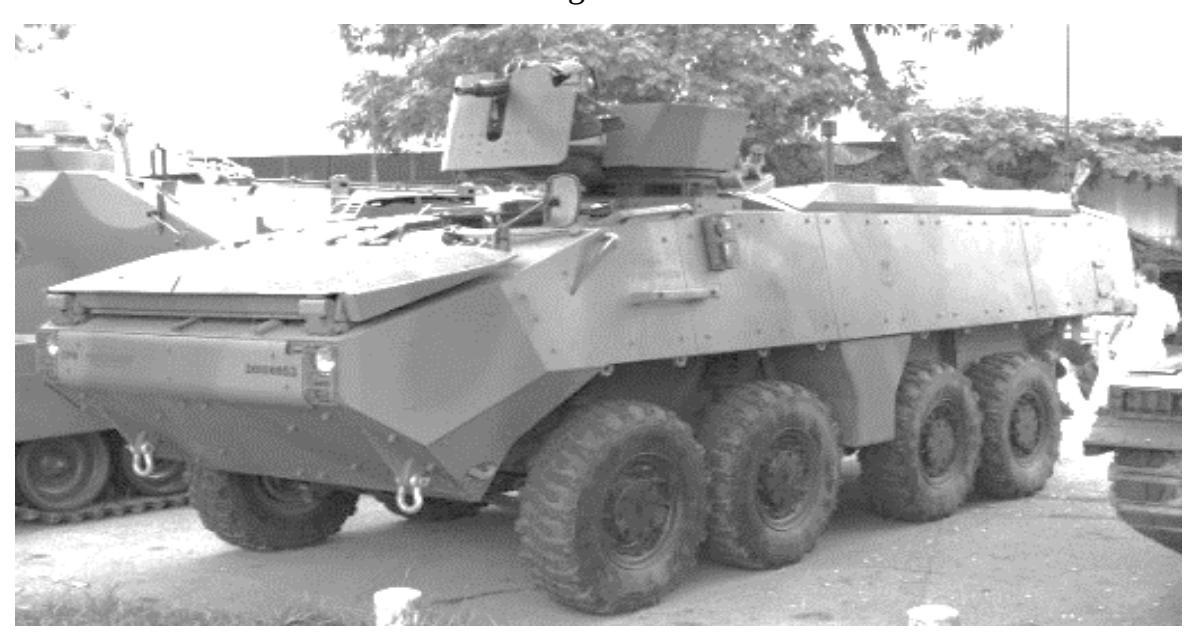

Fonte: Brasil (2008).

\subsubsection{CARRO SOBRE LAGARTA ANFÍBIO}

O Carro sobre Lagarta Anfíbio (CLAnf) é um veículo blindado, destinado ao transporte de pessoal, desenvolvido pela BAE Systems, Estados Unidos da América. Atualmente este carro anfíbio é empregado pelo CFN do Brasil e pelo United States Marine Corps (USMC) em desembarques anfíbios, dando todo suporte quanto à proteção e mobilidade aos Fuzileiros Navais. Seu tanque tem capacidade para 681 litros de diesel, proporcionando um raio de ação de $480 \mathrm{~km}$. Possui a capacidade total de transporte de 24 militares, sendo que três dessas vagas são para os operadores e 21 fuzileiros com seus equipamentos. Em termos de dimensão, sua blindagem é de $45 \mathrm{~mm}$, seu tamanho é de $8,10 \mathrm{x}$ 3,20 m (comprimento x largura), podendo obter uma velocidade máxima de $70 \mathrm{~km} / \mathrm{h}$. A Figura 5 dá uma visão geral do CLAnf.

Figura 5: Carro Lagarta Anfíbio (CLAnf).

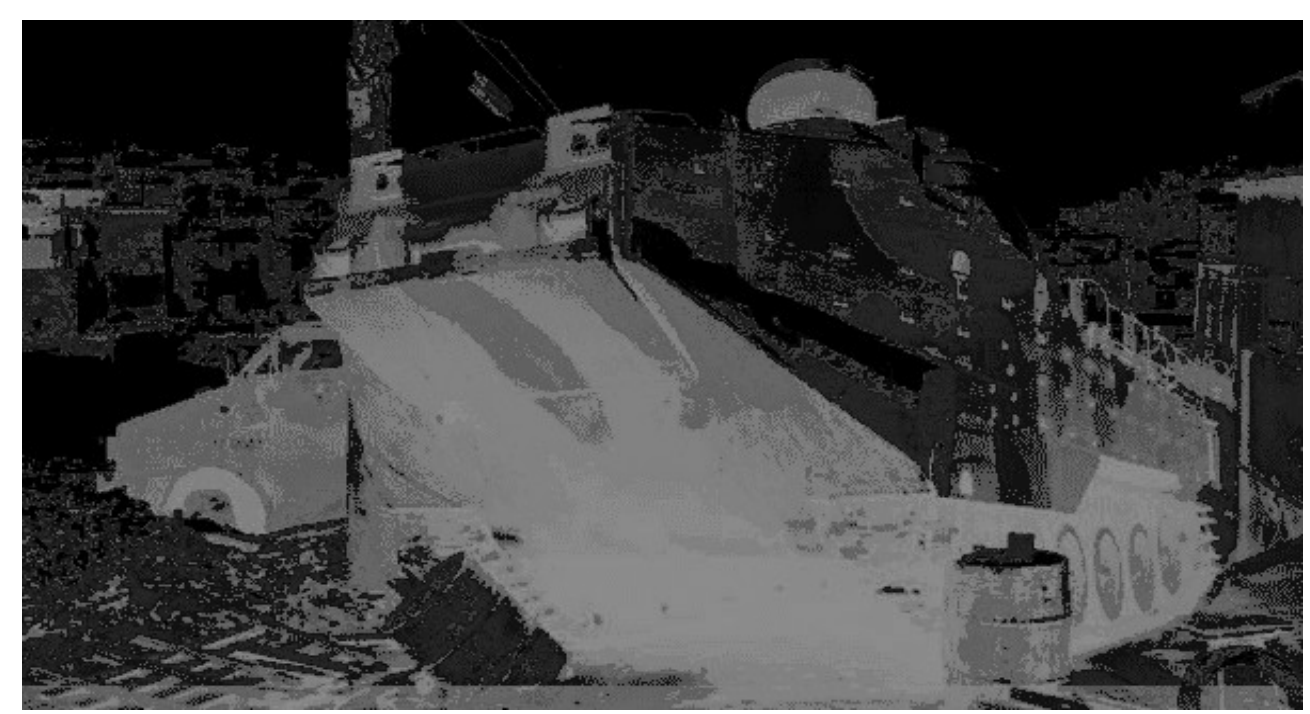

Fonte: Brasil (2008). 


\subsubsection{VIATURA BLINDADA M113-A1}

A viatura M113 - A1 faz parte de uma família de veículos blindados de transporte de pessoal de origem norte-americana. É um veículo sobre lagartas com capacidade anfíbia a pequenos cursos de água. Possui excelente capacidade de deslocamento em terreno e alta velocidade em estradas, sendo estas de asfalto ou terra batida. É uma viatura de transporte de tropa, onde leva um operador da viatura, um comandante de viatura e pode transportar até 11 passageiros armados e equipados no interior da viatura, totalizando 13 militares dentro da mesma. Seu tanque de combustível comporta 360L de diesel, com isso ele consegue um raio de ação de $480 \mathrm{~km}$, tendo como velocidade máxima $66 \mathrm{~km} / \mathrm{h}$. Em termos de dimensão, seu tamanho é de 4,86 x 2,68 m (comprimento x largura). Serve também como proteção do pessoal, contra disparos de armas de fogo contra os militares. A viatura também possui suporte para usabilidade de uma metralhadora .50. Na Figura 6 pode-se observar a viatura blindada M113 - A1.

Figura 6: M113-A1.

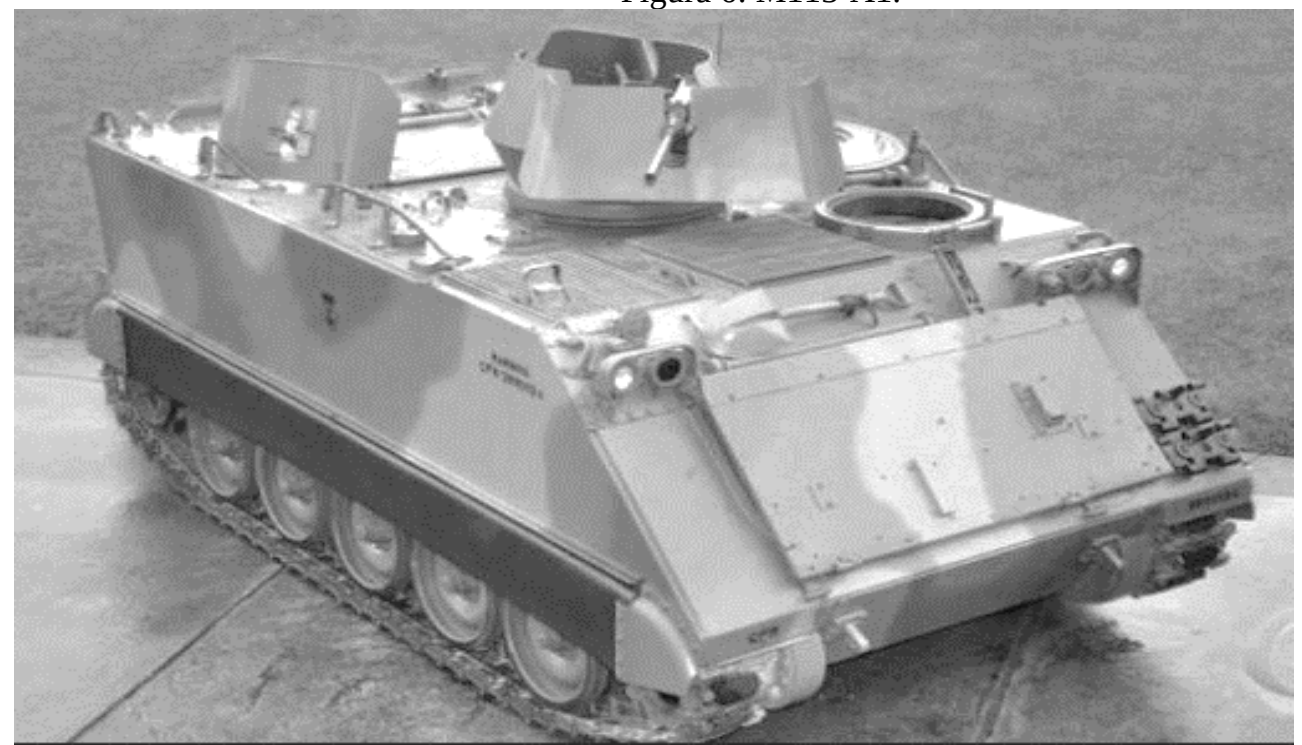

Fonte: Brasil (2008).

\subsection{CRITÉRIOS UTILIZADOS}

Com os modelos de carros apresentados, os critérios a serem avaliados para a evolução do trabalho são explicados a seguir:

- CONSUMO $(\mathrm{km} / \mathrm{l})$ : é importante porque as FFAA possuem um orçamento limitado. Assim, é desejável que o custo de operação seja o menor possível.

- AUTONOMiA (km): é extremamente importante que o veículo possa percorrer grandes distâncias sem a necessidade de reabastecimento constante. Esse ponto é crucial no momento de combate, pois permite que decisões possam ser tomadas com liberdade em momentos de tensão. 
- CAPACIDADE DE TRANSPORTE DE PESSOAL: é desejável que o veículo comporte o número de militares desejável para a missão. Desta forma, além do transporte do pessoal necessário, facilita a incursão nas comunidades através dos becos e vielas. Ou, ainda, pode ser utilizado para fazer resgate de pessoal.

- TIPO DE BLINDAGEM: é desejável que a blindagem do carro seja a mais robusta possível, de maneira a suportar os impactos de variados tipos de projetis, de maneira que os homens transportados pelo veículo não estejam expostos a um risco demasiadamente elevado.

- TAMANHO: O tamanho está relacionado diretamente ao ambiente em que o blindado será empregado, pois as comunidades do Rio de Janeiro contemplam ruas e vielas estreitas, onde veículos grandes encontram muita dificuldade em sua locomoção e para conseguirem adentrar com tranquilidade nos acessos da comunidade.

- MOBILIDADE: a velocidade e agilidade de locomoção das viaturas blindadas é muito importante, pois nas operações de GLO é necessário que as viaturas respondam de forma expedita, atendendo às peculiaridades deste tipo de missão.

O decisor 1 expressou as suas opiniões em relação aos critérios em ordenação de preferências, conforme consta na Tabela 1:

Tabela 1: Comparação paritária entre os critérios para o decisor 1

\begin{tabular}{|c|c|c|c|c|c|c|}
\hline & $\begin{array}{l}\text { Consumo } \\
\text { km/l }\end{array}$ & Autonomia & $\begin{array}{l}\text { Capacidade } \\
\text { de Pessoal }\end{array}$ & Blindagem & Tamanho & Mobilidade \\
\hline $\begin{array}{l}\text { Consumo } \\
\text { km/l }\end{array}$ & Equivalente & Muito Pior & Pior & Muito Pior & Pior & Pior \\
\hline Autonomia & $\begin{array}{l}\text { Muito Me- } \\
\text { lhor }\end{array}$ & Equivalente & Pior & Muito Pior & Melhor & Melhor \\
\hline $\begin{array}{l}\text { Capacidade } \\
\text { de Pessoal }\end{array}$ & Melhor & Melhor & Equivalente & Muito Pior & Pior & Muito Pior \\
\hline Blindagem & $\begin{array}{l}\text { Muito Me- } \\
\text { lhor }\end{array}$ & $\begin{array}{l}\text { Muito Me- } \\
\text { lhor }\end{array}$ & $\begin{array}{l}\text { Muito Me- } \\
\text { lhor }\end{array}$ & Equivalente & $\begin{array}{l}\text { Muito Me- } \\
\text { lhor }\end{array}$ & Melhor \\
\hline Tamanho & Melhor & Pior & Melhor & Muito Pior & Equivalente & Melhor \\
\hline Mobilidade & Melhor & Pior & $\begin{array}{l}\text { Muito Me- } \\
\text { lhor }\end{array}$ & Pior & Pior & Equivalente \\
\hline
\end{tabular}

Autores (2019)

Após o decisor 1 expressar suas opiniões, o decisor 2 expressou suas opiniões em relação aos critérios em ordenação de preferências, conforme consta na Tabela 2: 
Tabela 2: Comparação paritária entre os critérios para o decisor 2

\begin{tabular}{|c|c|c|c|c|c|c|}
\hline & $\begin{array}{l}\text { Consumo km/ } \\
\text { l }\end{array}$ & Autonomia & $\begin{array}{l}\text { Capacidade } \\
\text { de Pessoal }\end{array}$ & Blindagem & Tamanho & Mobilidade \\
\hline $\begin{array}{l}\text { Consumo } \\
\text { km/l }\end{array}$ & Equivalente & Pior & Muito Pior & $\begin{array}{l}\text { Absolutamente } \\
\text { Pior }\end{array}$ & Melhor & Muito Pior \\
\hline Autonomia & Melhor & Equivalente & Melhor & Muito Pior & Melhor & $\begin{array}{l}\text { Absolutamente } \\
\text { Melhor }\end{array}$ \\
\hline $\begin{array}{l}\text { Capacidade } \\
\text { de Pessoal }\end{array}$ & Muito Melhor & Pior & Equivalente & $\begin{array}{l}\text { Absolutamente } \\
\text { Pior }\end{array}$ & Pior & Muito Melhor \\
\hline Blindagem & $\begin{array}{l}\text { Absolutamente } \\
\text { Melhor }\end{array}$ & Muito Melhor & $\begin{array}{l}\text { Absolutamente } \\
\text { Pior }\end{array}$ & Equivalente & $\begin{array}{l}\text { Muito Me- } \\
\text { lhor }\end{array}$ & $\begin{array}{l}\text { Absolutamente } \\
\text { Melhor }\end{array}$ \\
\hline Tamanho & Muito Melhor & Pior & Melhor & Muito Pior & Equivalente & Melhor \\
\hline Mobilidade & Muito Melhor & $\begin{array}{l}\text { Absolutamente } \\
\text { Pior }\end{array}$ & Muito Pior & $\begin{array}{l}\text { Absolutamente } \\
\text { Pior }\end{array}$ & Pior & Equivalente \\
\hline
\end{tabular}

Autores (2019)

\subsection{DESENVOLVIMENTO DO MODELO}

Para o desenvolvimento do trabalho, dois militares foram entrevistados, desempenhando o papel de agentes decisores dentro do projeto. Nomeadamente, Capitão de Corveta Fuzileiro da MB, com curso de especialização e aperfeiçoamento do CFN e bastante experiência dentro da Frota. Cabo (FN), pertencente ao Batalhão de Blindados, com experiência em exercícios nacionais, sendo operador dos carros blindados. com vasto conhecimento técnico sobre esses blindados. Para iniciar o desenvolvimento do modelo, é apresentada a tabela com a escala de critérios utilizada (Tabela 3).

Tabela 3: Escala de critérios

\begin{tabular}{cc}
\hline Expressão Linguística Correspondente & Escala \\
\hline Absolutamente Pior & -3 \\
Muito Pior & -2 \\
Pior & -1 \\
Equivalente & 0 \\
Melhor & 1 \\
Muito Melhor & 2 \\
Absolutamente Melhor & 3 \\
\hline
\end{tabular}

Fonte: Teixeira, Santos e Gomes (2019)

Depois de conhecer os critérios a serem avaliados e a escala dos mesmos, os tomadores de decisão expressam suas opiniões sobre os critérios em ordem de preferência. Gerando desta forma um "peso" para cada critério.

\subsection{SAPEVOWEB}

A plataforma computacional para emprego do método SAPEVO-M denomina-se SapevoWeb e pode ser acessada pelo endereço: www.sapevoweb.com. Esta plataforma é um programa com uma interface gráfica amigável e apresenta resultados em tempo 
computacional reduzido (TEIXEIRA et al., 2018). O sistema SapevoWeb foi desenvolvido pelo Laboratório de Estudos de Governança, Gestão e Otimização (LEGGO), a partir de uma parceria entre o corpo técnico do Centro de Análise de Sistemas Navais (CASNAV), um grupo de pesquisa do Programa de Pós-Graduação em Engenharia de Produção da Universidade Federal Fluminense (UFF) e um grupo de pesquisa do Programa de PósGraduação em Engenharia de Sistemas e Computação do Instituto Militar de Engenharia (IME).

Foi criado com o objetivo de compilar os dados fornecidos pelos decisores, sendo responsável por todo o processamento matemático, dando como retorno o resultado da ordenação das alternativas, da globalmente melhor para a globalmente pior. O sistema funciona da seguinte maneira:
$\checkmark \quad 1^{\circ}$ passo: o usuário cadastra o nome do projeto;
$\checkmark \quad 2^{\circ}$ passo: o usuário cadastra os decisores;
$\checkmark \quad 3^{\circ}$ passo: o usuário cadastra as alternativas;
$\checkmark \quad 4^{\circ}$ passo: o usuário cadastra os critérios;
$\checkmark \quad 5^{\circ}$ passo: os decisores avaliam os critérios, em ordem de preferência;
$\checkmark \quad 6^{\circ}$ passo: os decisores avaliam as alternativas, em relação a cada critério;
$\checkmark \quad 7^{\circ}$ passo: obtém-se o resultado dos pesos em relação aos critérios; e
$\checkmark \quad 8^{\circ}$ passo: obtém-se o resultado da ordenação das alternativas.

A partir da compilação dos dados na base SapevoWeb, o Software gera os pesos de cada critério. Os resultados obtidos por meio do SapevoWeb em relação aos pesos estão descritos na Tabela 4: 


\begin{tabular}{ll} 
Consumo km/l & 0003 \\
\hline Autonomia & 1.108 \\
\hline Capacidade pessoal & 0.641 \\
Blindagem & 2.0 \\
\hline Tamanho & 0.665 \\
Mobilidade & 0.817 \\
\hline
\end{tabular}

Fonte: Autores (2019)

Como pode ser observado na Tabela 4, o critério blindagem é o de maior peso, pois em uma incursão em comunidade ou situações que se exigem blindados é o critério que estabelece segurança aos envolvidos no decorrer da missão. O critério com o segundo maior peso foi da autonomia, por este critério permitir que veículos blindados possam ter um raio de ação maior nas missões, garantindo a permanência no terreno. $\mathrm{O}$ critério com o terceiro maior peso foi a mobilidade. Observa-se também a importância deste critério nas atividades operacionais das FFAA, pois este critério garante uma maior agilidade nas atividades, podendo resgatar feridos com mais rapidez. Cabe ressaltar que poucos minutos a mais no resgate ou reforço faz toda diferença, podendo obter êxito na missão e até mesmo salvar uma vida. O quarto maior peso foi estipulado para o critério tamanho, pois vale salientar que em casos que viaturas blindadas são exigidas em uma operação em comunidade, tem que se ter em vista as dificuldades que o mesmo irá enfrentar, como ruas estreitas e muitos obstáculos para a transição. O quinto maior peso é a capacidade de pessoal, que está relacionada à quantidade que uma viatura blindada pode transportar. Vale ressaltar que não basta um veículo blindado comportar uma quantidade muito elevada, se ele não conseguir ser eficaz nas incursões de GLO. E por último o de menor peso foi o consumo, pois embora a economia de recursos financeiros seja importante, não pretere à salvaguarda da vida humana e o sucesso da missão.

\section{RESULTADOS}

A viatura blindada Piranha IIIC é o veículo que se destacou entre os demais, em relação aos critérios estabelecidos, tendo em vista que ele se destaca em relação a autonomia que tem o segundo maior peso e mobilidade que tem o terceiro maior peso. No critério blindagem, o Piranha IIIC perde para o CLAnf, porém ganha do M113-A1 e se equipara ao Sk-105 A2S. Além disso, no quesito blindagem ele fica em segundo lugar, tendo em vista que é o critério que tem o maior peso. Já em relação ao tamanho, ele perde apenas para o M113-A1 e ganha do Sk-105 A2S e do CLAnf. Sua capacidade de transporte de pessoal é de 2+12 homens, perdendo apenas para o CLAnf que leva 3+21 homens e ganha do Sk-105 A2S que transporta apenas a tripulação de 3 pessoas e do M113-A1 que leva 2+11 homens. Contudo, o Piranha IIIC perde para todos os outros carros blindados no critério consumo $\mathrm{Km} /$ Litro. O veículo Piranha IIIC ganha o $1^{\circ}$ lugar pelo motivo de possuir a vantagem entre os demais veículos em dois critérios que tem os maiores pesos dentro das características de operacionalidade em missões especiais de GLO, fazendo assim dele o vencedor, dentre as quatros alternativas propostas. 
Tabela 5: Resultado

\begin{tabular}{l}
\hline \multicolumn{1}{c}{ Ordenação do Resultado } \\
\hline $1^{\circ}$ Piranha IIIC $\rightarrow 5.989$ \\
$2^{\circ}$ CLAnf $\rightarrow 5.970$ \\
$3^{\circ}$ Sk-105 A2S $\rightarrow 3.191$ \\
$4^{\circ}$ M113-A1 $\rightarrow$ 1.663 \\
\hline
\end{tabular}

Fonte: Autores (2019)

\section{CONCLUSÃO}

Este estudo evidenciou, dentre as principais viaturas blindadas da Marinha do Brasil, a partir de critérios práticos, qual a melhor opção para missões de GLO. Cabe ressaltar a redução de um eventual desperdício de tempo e recursos em relação a escolha de blindados, graças à utilização de uma técnica de Apoio Multicritério à Decisão. Antes de se iniciar o projeto, foi necessária a compreensão das práticas executadas no CFN, para o entendimento sobre as possíveis utilizações dos blindados nas missões.

Dentre os métodos multicritérios pesquisados para a solução deste problema real, verificou-se a adequação do método multicritério SAPEVO-M, de fácil utilização por meio do Software SapevoWeb, que tem a finalidade de processar toda a parte matemática. A ferramenta SapevoWeb possibilitou a ordenação dos blindados, sendo estes classificados do mais preferível para o menos preferível, de modo que numa eventualidade da melhor alternativa não estar disponível por qualquer motivo, pode-se escolher a melhor viatura subsequente.

Os decisores, a partir das suas experiências em zonas conflagradas, expressaram as suas opiniões com a visão de onde os blindados possivelmente poderão ser empregados, com olhar crítico ao ambiente que ele será introduzido, como também as possíveis ameaças, para assim poderem expressar suas preferências de acordo com cada caso.

Os resultados obtidos por meio da ferramenta evidenciam os benefícios obtidos com o projeto, pois com a sua implantação é possível alcançar os objetivos operacionais, como também contribuir com a sociedade brasileira que tanto sofre com a violência nos grandes centros urbanos. Sendo assim, o processo de estruturação e modelagem matemática apresentados nessa pesquisa foram considerados adequados, na medida que foi possível ranquear, do melhor para o pior, os veículos blindados utilizados pelo Corpo de Fuzileiros Navais em operações de Garantia da Lei e da Ordem (GLO).

\section{REFERÊNCIAS BIBLIOGRÁFICAS}

[1] ARENAleS, M.; ARMENTANO, V.; MORABITO, R.; YANASSE, H. Pesquisa operacional. Rio de Janeiro: Elsevier, p.524, 2007.

[2] BRANS, J. P., \& MARESCHAL, B. (2005). Multiple criteria decision analysis state of the art.Dordrecht: Kluwer Academic Publishers.

[3] BRASIL. Comando Geral do Corpo de Fuzileiros Navais. Manual de Operações Anfíbias dos Grupamentos Operativos de Fuzileiros Navais, 1 ${ }^{\text {a }}$ Ed. 2008.

[4] BRASIL. Constituição da República Federativa do Brasil. Brasília, DF: Senado Federal: Centro Gráfico, 1988. 
[5] Carro Lagarta Sk-105 A2S. Disponível em: $<$ http://minhamarinha.blogspot.com/2014/02/cfn-carro-lagarta-sk-105-marinhado.html/>. Acesso em 24 de março de 2019.

[6] Carro Piranha IIIC. Disponível em: $<$ http://www.planobrazil.com/2016/05/08/adsumusviatura-blindada-especial-sobre-rodas-vtrbldespsr-8x8-piranha-iiic/>. Acesso em 24 março de 2019.

[7] Carro Lagarta Anfíbio. Disponível em: $<$ https://www.warfareblog.com.br/2018/08/baesystems-aav-7-o-rei-da-praia.html >. Acesso em: 26 de março de 2019.

[8] Carro M-113 A1. Disponível em: $<\underline{\text { http://www.operacional.pt/m113-o-cavalo-de- }}$ batalha-da-infantaria-mecanizada/>. Acesso em 28 de mar.2019.

[9] DE ALMEIDA, Adiel Teixeira. Processo de decisão nas organizações: construindo modelos de decisão multicritério. São Paulo: Atlas, 2013.

[10] Emenda Constitucional $\mathrm{n}^{\circ} 99$ de 14 de dezembro de 2017, Art. 142. Disponível em: $<$ https://www.senado.leg.br/atividade/const/con1988/CON1988 05.10.1988/ art_142_asp $>$. Acesso em 24 de março de 2019.

[11] GOMES, C.F.S.; MAIA, A. C. C. (2012). Biomassa como alternativa para o fornecimento de energia. Pesquisa Operacional para o Desenvolvimento, v.4, n.1.

[12] GOMES, L. F. A. M.; MURY, A. R.; GOMES, C. F. S. Multicriteria Ranking with Ordinal Data. Systems Analysis, Modelling, Simulation (Print), v. 27 , p. 139-146, 1997.

[13] GOMES, L. F. A. M.; GOMES, C. F. S. (2019). Princípios e métodos para a tomada de decisão: Enfoque multicritério (6a ed.). São Paulo: Atlas.

[14] HILLIER, Frederick S., LIEBERMAN, Gerald J. Introdução à Pesquisa Operacional. São Paulo/SP, McGraw Hill, 2006.

[15] MARINS, Fernando Augusto Silva. Introdução à Pesquisa Operacional. São Paulo: Cultura Acadêmica: Universidade Estadual Paulista, Pró-Reitoria de Graduação, 2011.

[16] SOBRAPO. Disponível em: < https://www.sobrapo.org.br/>. Acesso em 26 de março de 2019.

[17] TEIXEIRA, L. F. H. S. B., SANTOS, M. e GOMES, C. F. S. SapevoWeb Software (v.1). (2018). Disponível em: http://www.sapevoweb.com. Acesso em: 20/06/ 2019.

[18] TEIXEIRA, Luiz Frederico Horácio de Souza de Barros; SANTOS, Marcos dos; GOMES, Carlos Francisco Simões. Proposta e implementação em python do método Simple Aggregation of Preferences Expressed by Ordinal Vectors - Multi Decision Makers: uma ferramenta web simples e intuitiva para Apoio à Decisão Multicritério. In: SIMPÓSIO DE PESQUISA OPERACIONAL E LOGÍSTICA DA MARINHA, 19., 2019, Rio de Janeiro, RJ. Anais [...]. Rio de Janeiro: Centro de Análises de Sistemas Navais, 2019. 